\title{
Laser-vacuum deposited ITO thin films for optoelectronic applications
}

\author{
A S Toikka ${ }^{1,2,3}$ and N V Kamanina ${ }^{1,2,3}$ \\ ${ }^{1}$ Lab for Photophysics of media with nanoobjects, Vavilov State Optical Institute, \\ 199053 St. Petersburg, Russia \\ ${ }^{2}$ Department of Photonics, St. Petersburg Electrotechnical University ("LETI"), \\ 197376 St. Petersburg, Russia \\ ${ }^{3}$ Advanced Development Division, Petersburg Nuclear Physics Institute, 188300 \\ Gatchina, Russia \\ Corresponding author: A S Toikka; e-mail: atoikka@obraz.pro
}

\begin{abstract}
In the current paper, it has been considered the relief features and electric properties of the transparent contacts based on the indium tin oxides. During the formation of samples, the laser oriented deposition method using the $\mathrm{CO}_{2}$ laser operated at the wavelength of $10.6 \mu \mathrm{m}$ and with the power of $30 \mathrm{~W}$ has been used. The deposition process has been revealed via varied the intensity of external electric field in the range of $100-600 \mathrm{~V} / \mathrm{cm}$. The influence of the electric field on the deposition process and its correlation with the relief features and electric properties have been evaluated.
\end{abstract}

Keywords. Laser-vacuum deposition technique, indium tin oxides, transparent contacts, antireflective coatings, waterproof covering.

\section{Introduction}

Indium tin oxides (ITO) is a degenerate semiconductor with $n$-type of conductivity. Due to the high optical transmittance in the visible and near-infrared (NIR) spectral ranges and low sheet electrical resistance, ITO thin films perform functions of transparent contacts in the constructions of electrooptical modulation devices [1][2][3], solar cells [4][5], sensors [6][7], etc.

For the development of modulation devices, as optical limiters, wavefront correctors, spatial-light modulators, and other components based on ITO [8][9][10][11], high transmittance level and small signal-to-noise ratio are required. In this case, one of the main issues for the device development is to decrease the reflection losses on the interface of ITO with the neighbor layers. It is also important for index-matching of transparent conducting films with photo absorber layers during solar cell fabrication [12][13]. Moreover, thermal and electric stability of films are the commonly required parameters as well. In order to control the basic properties of ITO, various technological steps are used, such as switching the parameters during the deposition, thermal treatment, structurization with nanoparticles and laser processing [14][15][16]. 
Unfortunately, the quantity of indium is finite in the Earth core, so the cost of films based on this material is gradually increasing. Partially it could be solved via the use of alternative compositions, for example, Al-doped zinc oxides (AZO) [17] and fluorine-doped tin oxides (FTO) [18]. They also have small optical losses in the bulk. However, the non-optimized surface of transparent oxides leads to high reflectance. Due to this aspect, ITO thin films are still the key material for the wide quantity of configurations for the liquid crystal devices and solar cells.

Depending on issues, application area and production scale, the various methods for deposition of ITO can be used. Magnetron sputtering and chemical vapor deposition from the gas phase are the methods for mass-scale fabrication. However, due to the limitations with the orienting of deposited particles in these methods, the relative heterogeneity in relief exists. The atomic layer deposition (ALD) technique performs the fabrication of the smooth and homogeneous ITO films [19]. However, the scale of fabrication is limited by the cost of equipment. It should be noticed, that technologies based on laser deposition have practice interest on account of few reasons. Firstly, deposition flux will depend on the laser power density, frequency, and processing velocity what permits to vary the electro-optical properties of ITO films. Secondary, the deposited particles can be controlled via the additional source of an electric field. The laser oriented deposition (LOD) approach is suitable for mass-scale fabrication, so it could be a compromise between the high-precise ALD technique [20][21] and scalable PVD [22][23] and CVD [24] methods.

In this paper, we will consider the electric properties and the relief features of laser-vacuum deposited ITO thin films with the applying of an additional electric field in order to orient the deposited particles. Laser oriented deposition technique leads to a limit of indium consumption and increase the application area of ITO.

\section{Materials and methods}

The Cerac Inc. powder with indium tin oxide (90-10 wt $\%, 3-12 \mathrm{~mm} \mathrm{pcs}, 99.99 \%$ pure) was the material source. Laser oriented deposition scheme consists of $\mathrm{CO}_{2}$ laser $(\lambda=10.6 \mu \mathrm{m})$, an opticmechanical system for beam parameters setting (quasi-continuous regime, power $30 \mathrm{~W}$, beam diameter $5 \mathrm{~mm}$, processing velocity $\sim 3 \mathrm{~cm} \times \mathrm{c}^{-1}$ ) and vacuum post (pressure $10^{-1} \mathrm{~Pa}$ before the deposition). For additional control of film deposition, the conductive grid (named on Figure 1 as electric supply system) was installed inside the vacuum chamber. For the different samples, the various electric field was applied: $0 \mathrm{~V} \times \mathrm{cm}^{-1}, 100 \mathrm{~V} \times \mathrm{cm}^{-1}, 200 \mathrm{~V} \times \mathrm{cm}^{-1}$, and $600 \mathrm{~V} \times \mathrm{cm}^{-1}$ respectively. The thickness of films is $100 \mathrm{~nm}$ on $20 \mathrm{~mm} \times 20 \mathrm{~mm} \times 2 \mathrm{~mm}$ glass substrates.

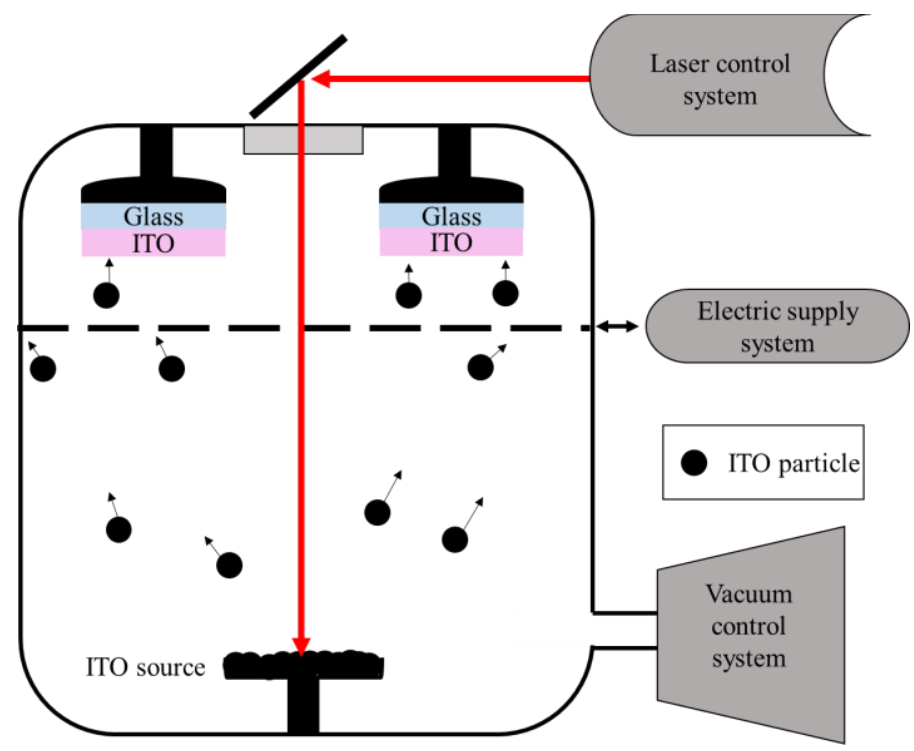

Figure 1. Laser oriented deposition schematic diagram 
Atomic-force microscope Solver Next (NT-MDT) used for the relief characterization (method of constant force, scan area $30 \mu \mathrm{m} \times 30 \mu \mathrm{m}$, scan rate $1 \mathrm{~Hz}$ ). The setting of parameters and data processing were performed in Nova Px software. Wetting angle optic system OCA 15 EC (DataPhysics Instruments $\mathrm{GmbH}$ ) used for the ITO surface diagnostic in macro-scale. Distillate water drops with 1 $\mu \mathrm{l}$ volume consequently deposited per the full aperture of the sample surfaces with CCD camera recording. Calculation of wetting angle was performed via the computer vision technique and LaplaceYoung approximation in SCA20 software. Electric properties were measured via the 2-point measurement scheme with GW Instek PPE-3323 power supply, Tektronix AFG 3021B pulse generator, Rigol DS1102D oscilloscope and Fluke 8846A multimeter.

\section{Results and discussions}

Electric field during the ITO deposition influences the particle's velocity and corrects their direction relative to the substrate.

For relief statistic description average roughness $\left(S_{a}\right)$ and average root-mean-square roughness $\left(S_{q}\right)$ were used:

$$
\begin{gathered}
S_{a}=\frac{\sum_{i=1}^{n} \sum_{j=1}^{m}\left(z_{i, j}\right)}{n m} \\
S_{q}=\sqrt{\frac{\sum_{i=1}^{n} \sum_{j=1}^{m}\left(z_{i, j}\right)^{2}}{n m}}
\end{gathered}
$$

Where $i$ and $j$ indices correspond to $x$ and $y$ axis respectively $(n=256, m=256) ; z_{i, j}$ - height for point with $(i, j)$ coordinates. The examples of areas for the different ITO deposition conditions are demonstrated in Figure 2.

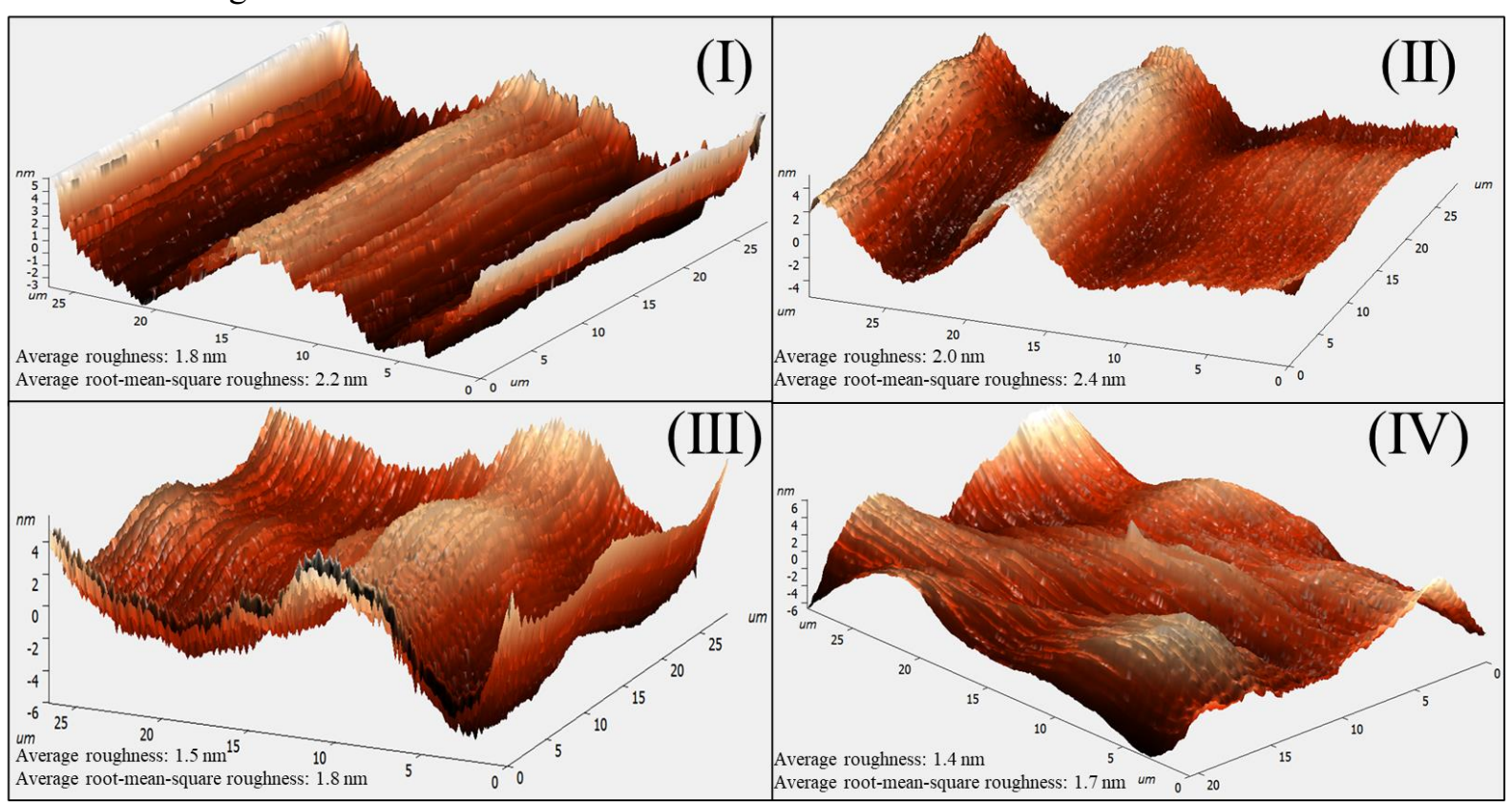

Figure 2. Atomic force microscopy relief characterization of pure ITO films deposited under different electric fields: $0 \mathrm{~V} / \mathrm{cm}$ (I), $100 \mathrm{~V} / \mathrm{cm}$ (II), $200 \mathrm{~V} / \mathrm{cm}$ (III) and $600 \mathrm{~V} / \mathrm{cm}$ (IV)

The relief of the ITO films represents the layer-by-layer deposition with island growth formation (Stranski-Krastanov mechanism). The values of $S_{a}$ and $S_{q}$ are given as average value for 5 areas per each technological mode. With using fields of $200 \mathrm{~V} / \mathrm{cm}$ and $600 \mathrm{~V} / \mathrm{cm}$ the relief becomes smoother. In these cases, deposited particles have enough energy to take positions in surface irregularities. 
The geometry of the surface and its compositional consist influence on the free volume energy, which can visualize via the wetting angle measurements. For each sample consequently deposited distilled water and measured wetting angle per full aperture (Figure 3).

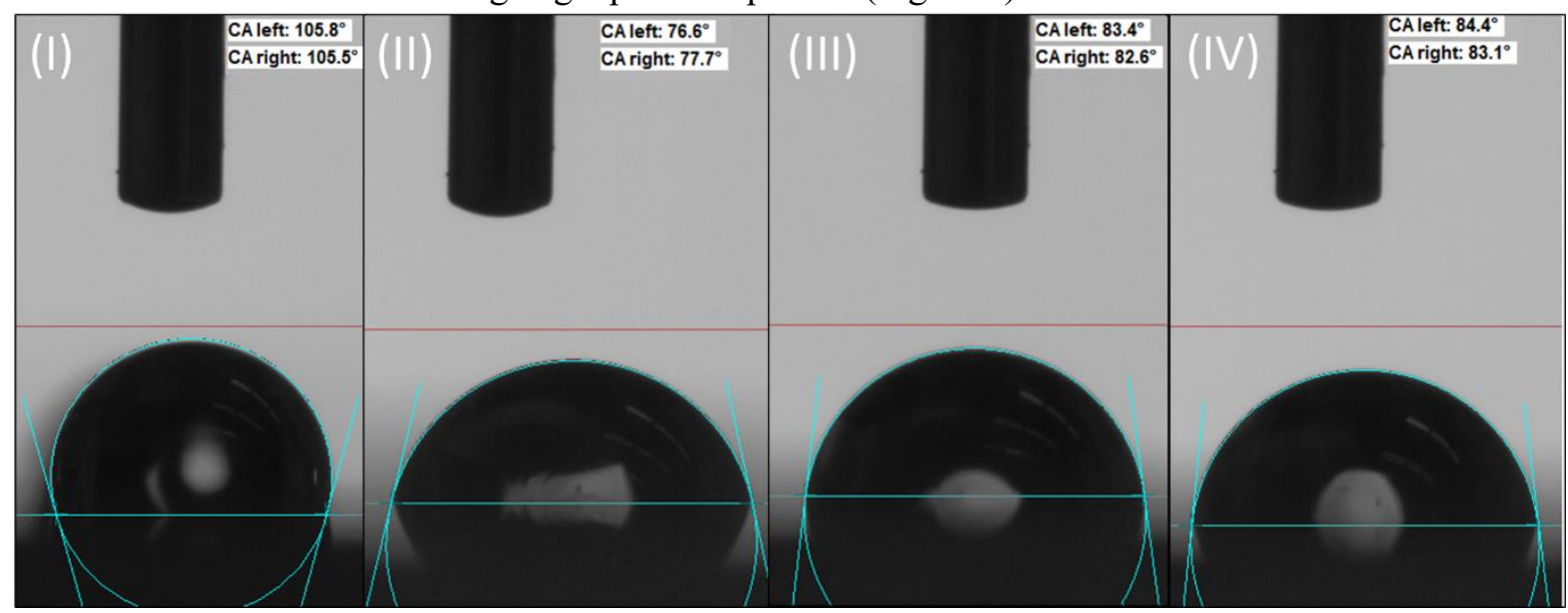

Figure 3. Wetting angle measurement images of pure ITO films deposited under different electric fields: $0 \mathrm{~V} / \mathrm{cm}$ (I), $100 \mathrm{~V} / \mathrm{cm}$ (II), $200 \mathrm{~V} / \mathrm{cm}$ (III) and $600 \mathrm{~V} / \mathrm{cm}$ (IV)

The high influence on the wetting angle gives surface tension that depends on the contact area. The dependency of wettability versus roughness is non-linear. The surface irregularity produces a higher «liquid-ITO» contact area and leads to rising in the wettability (the wetting angle becomes lower). However, if the distances between heterogeneities are much less than drop sizes, this massive have water-proof properties (wetting angle rises). Due to these reasons, the ITO films with the biggest roughness $(E=100 \mathrm{~V} / \mathrm{cm})$ have better wettability in comparison with other samples. In the case of deposited ITO without field, the relief has small distances between heterogeneities, which leads to an increase of wetting angle (Figure 4).

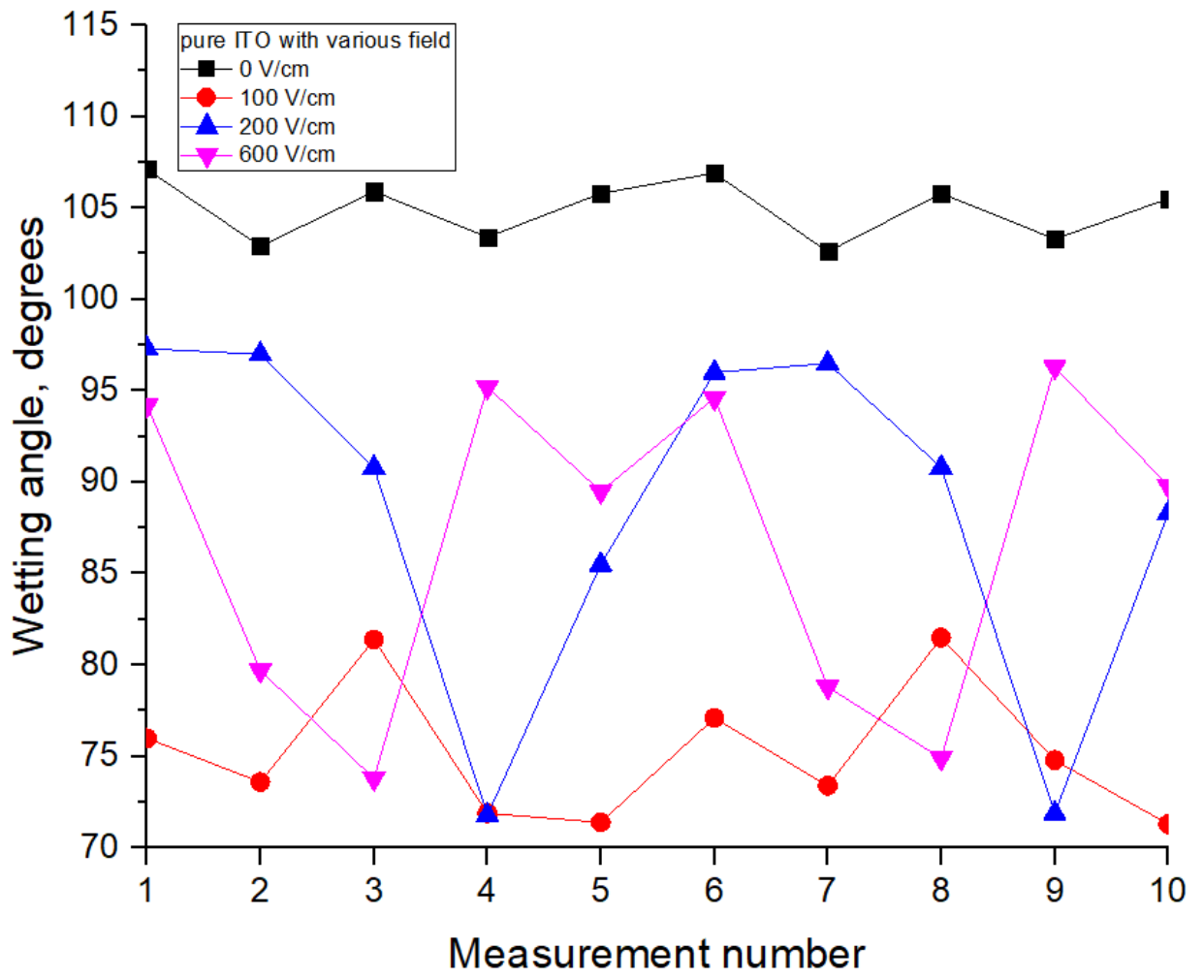

Figure 4. Wetting angle statistics of the pure ITO films deposited under different electric fields 
It should be mentioned, that the optical modulation devices, which operate in the wide commutation frequency range, require the ITO films stability of electrical properties. To degenerate the pure ITO, the main charge transfer channel is the surface. So, the resistance of ITO films is limited by the number of defects near the surface. For impedance measurements, we used the liquid gallium metallization. Full resistance of samples consisted of the ITO resistance $R_{\text {ITO }}$ with capacity $C_{\text {ITO }}$ in parallel, with contact resistance $R_{I T O-G a}$ and metal inductance $L_{G a}$ in series. The measurements under the digital current show a decrease of the resistance for the deposited ITO with the electric field applying (Figure 5). The amplitude for digital current and analog current for impedance measurements was equal to $5 \mathrm{~V}$.

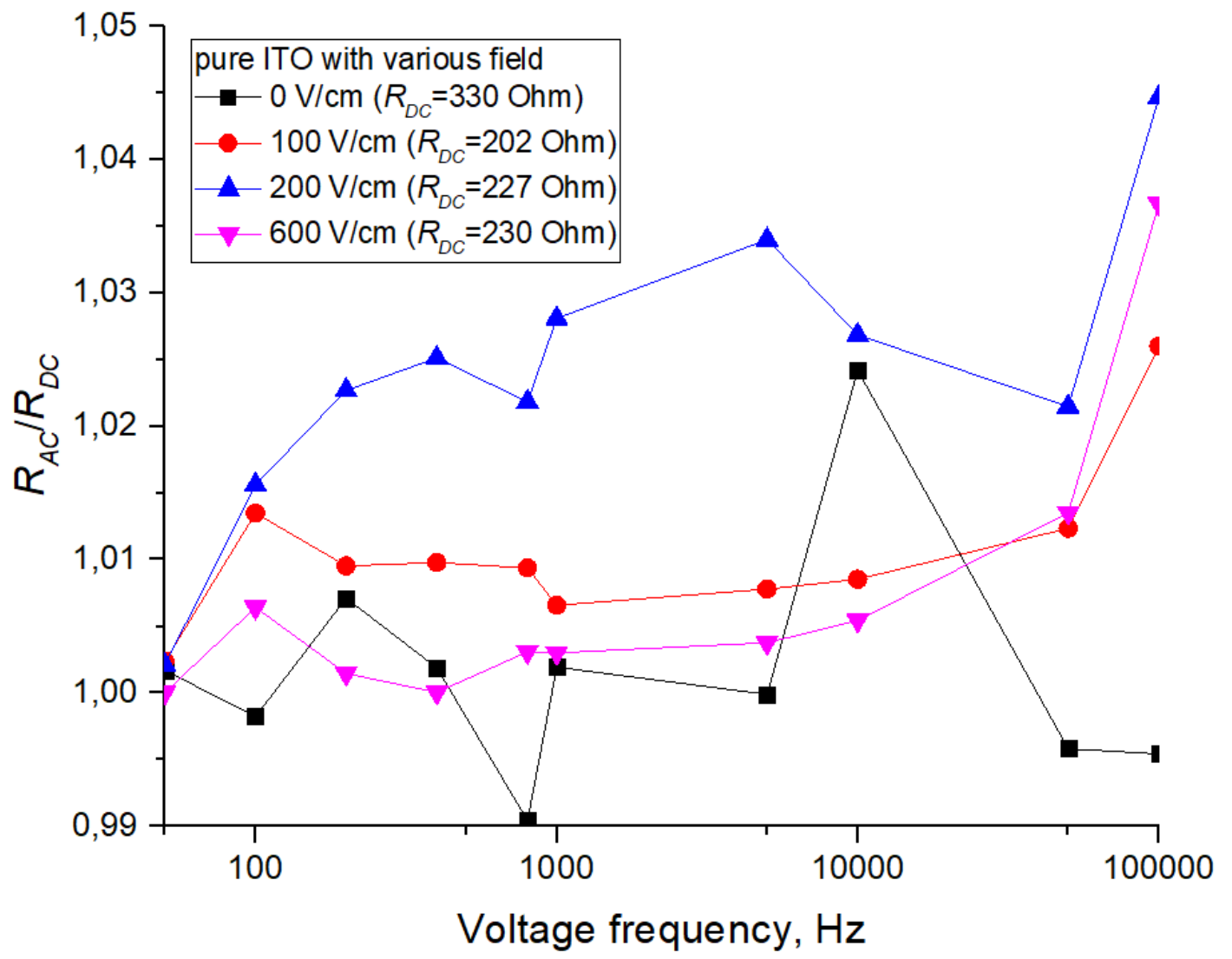

Figure 5. Impedance frequency dependence of pure ITO deposited under the various electric fields

In frequency range from $50 \mathrm{~Hz}$ to $50 \mathrm{kHz}$ the ITO films have stable impedance values (the fluctuations don't exceed the experimental setup error). Impedance increases by more than $1.5 \%$ when $f=100 \mathrm{kHz}$ for the ITO films deposited with the field. This phenomenon is connected with the rise of inductance reactive resistance. For the ITO film without an electric field use during deposition, the impedance doesn't change dramatically, due to the compensation of the capacity and an inductance resistance in the observed frequency range. ITO capacity has a negative impact on temporal characteristics, for example, switching time, of modulation devices.

\section{Conclusion}

To summarize the obtained results, one can conclude the following aspects. The application of an additional electric field in the laser-vacuum deposition technique allows controlling the relief and electrical properties of the pure ITO films. With using an electric field of $200 \mathrm{~V} / \mathrm{cm}$ and of $600 \mathrm{~V} / \mathrm{cm}$ during the deposition, the ITO samples have less roughness, namely as $1.5 \mathrm{~nm}$ and $1.4 \mathrm{~nm}$ respectively, against $1.8 \mathrm{~nm}$ against for an electric field of $0 \mathrm{~V} / \mathrm{cm}$. This approach is useful for the 
further deposition of nanoobjects, for example, carbon nanotubes or the reduced graphene oxides, or other functional layers in devices because the interface will be more uniform.

In the case of fabrication of the waterproof optical transparent coatings based on pure ITO, the mode without an electric field use is more suitable (the average wetting angle is near 105 degrees). However, in order to decrease the wettability, ITO can perform the function of the buffer layer for nanoparticles. For these issues, the surface of the ITO should be smoothest, which requires the application of an electric field during the deposition.

Pure ITO has different electrical properties. Deposited films with using of electric fields have less resistance (200-230 Ohm in comparison with $330 \mathrm{Ohm}$ ). Moreover, according to impedance frequency dependencies, this approach allows neglecting the capacity influence in the range from $50 \mathrm{~Hz}$ to 100 $\mathrm{kHz}$. In ITO deposited without an electric field, metallization inductance is compensated by film capacity. As a result, the use of electric field during the ITO deposition allows neglect of negative impact of capacity on the temporal characteristics of modulation devices.

\section{Acknowledgments}

The authors would like to thank the colleagues from the Photophysics lab (Vavilov State Optical Institute, St. Petersburg) and to commend their colleagues from Nuclear Physics Institute for help in work and for perfect discussions.

\section{References}

[1] R. Amin, R. Maiti, J.K. George, X. Ma, Z. Ma, H. Dalir, M. Miscuglio, V.J. Sorger: A lateral MOS-Capacitor-Enabled ITO Mach-Zehnder Modulator for Beam Steering. J. of Lighwave Technology, 38(2), 282-290 (2020)

[2] A.S. Toikka, N.V. Kamanina: Liquid crystals electro-optical structure with conducting layers modified by carbon nanotubes. J. of Physics: Conference Series, 1695(012071), 1-4 (2020)

[3] S. Rajput, V. Kaushik, S. Jain, P. Tiwari, A.K. Srivastava, M. Kumar: Optical Modulation in Hybrid Waveguide Based on Si-ITO Heterojunction. J. of Lighwave Technology, 38(6), 1365-1371 (2020)

[4] S. Lu, Y. Sun, K. Ren, K. Liu, Z. Wang, S. Qu: Recent Development in ITO-free Flexible Polymer Solar Cells. Polymers, 10(5), 1-30 (2018)

[5] J.H. Kim, H.J. Seok, H.J. Seo, T.Y. Seong, J.H. Heo, S.H. Lim, K.J. Ahn, H.K. Kim: Flexible ITO films with atomically flat surfaces for high performance flexible perovskite solar cells. Nanoscale, 44, 20587-20598 (2018)

[6] C. Liu, J. Wang, F. Wang, W. Su, L. Yang, J. Lv, G. Fu, X. Li, Q. Liu, T. Sun, P.K. Chu: Surface plasmon resonance (SPR) infrared sensor based on D-shape photonic crystal fibers with ITO coatings. Optics Communications, 464 (125496), 1-8 (2020)

[7] M. Śmietana, M. Koba, P. Sezemsky. K. Szot-Karpińska, D. Burnat, V. Stranak, J. NiedziółkaJönsson, R. Bogdanowicz: Simultaneous optical and electrochemical label-free biosensing with ITO-coated lossy-mode resonance sensor. Biosensors and Bioelecntronics, 154 (112050), 1-7 (2020)

[8] L. Begel, T. Galstian: Liquid crystal lens with corrected wavefront asymmetry. Applied Optics, 57(18), 5072-5078 (2018)

[9] N.V. Kamanina, Yu. A. Zubtsova, A.A. Kukharchik, C. Lazar, I. Rau: Control of the IR-spectral shift via modification of the surface relief between the liquid crystal matrixes doped with the lanthanide nanoparticles and the solid substrate. Optics Express, 24(2), 270-275 (2016)

[10] J. Zou, Q. Yang, E.L. Hsiang, H. Ooishi, Z. Yang, K. Yoshidaya, S.T. Wu: Fast-Response Liquid Crystal for Spatial Light Modulator and LiDAR Applications. Crystals, 11(93), 1-10 (2021)

[11] N.V. Kamanina, Y. A. Zubtsova, A.S. Toikka, S.V. Likhomanova, A. Zak, R. Tenne: Temporal Characteristics of Liquid Crystal Cell with $\mathrm{WS}_{2}$ nanoparticles: Mesophase sensitization and relief features. Liq. Cryst. And their Appl., ISSN 1991-3966, 20(1), 34-40 (2020) 
[12] K. Bittkau, T. Kirchartz, U. Rau: Optical design of spectrally selective interlayers for perovskite/silicon heterojunction tandem solar cells. Optics Express, 26(18), 750-760 (2018)

[13] Y. Li, J.D. Lin, X. Liu, Y. Qu, F.P. Wu, F. Liu, Z.Q. Jiang, S.R. Forrest: Near-Infrared Ternary Tandem Solar Cells. Advanced Materials, 30(1804416), 1-20 (2018)

[14] B.B. Sahu, L. Wen, S.H. Kim, J.G. Han: Study of plasma characteristic and properties of flexible ultra-thin ITO films prepared by large area 3-D confined and planar magnetron sputtering. Vacuum, 165, 246-253 (2019)

[15] N. Xia, R.A. Gerhardt: Fabrication and characterization of highly transparent and conductive indium tin oxide films made with different solution-based methods. Materials Research Express, 3(116408), 1-11 (2016)

[16] N. Kamanina, A. Toikka, I. Gladysheva: ITO conducting coatings properties improvement viananotechnology approach. Nano Express, 2(010006), 1-7 (2021)

[17] I. Jellal, H. Ahmoum, Y. Khaaissa, K. Nouneh, M. Boughara, M. Fahoume, S. Chopra, J. Naja: Experimental and ab-initio investigation of the microstructure and optoelectronic properties of FCM-CVD-prepared Al-doped ZnO thin films. Applied Physics A, 125(650), 1-7 (2019)

[18] D. Zhang, H. Tian, S. Bu, T. Yan, J. Ge, T. Lei, W. Bi, L. Huang, Z. Ge: Efficient planar heterojunction perovskite solar cells with enhanced $\mathrm{FTO} / \mathrm{SnO}_{2}$ interface electronic coupling. J. of Alloys and Compounds, 831(154717), 1-9 (2020)

[19] J.A. Libera, J.N. Hryn, J.W. Elam: Indium Oxide Atomic Layer Deposition Facilitated by the Synergy between Oxygen and Water. J. Chem. Mater, 23(8), 2150-2158 (2011)

[20] B. Zhao, M. Nisula, A. Dhara, L. Henderick, F. Mattelaer, J. Dendooven, C. Detavernier: Atomic Layer Deposition of Indium-Tin-Oxide as Multifunctional Coatings on $\mathrm{V}_{2} \mathrm{O}_{5}$ ThinFilm Model Electrode for Lithium-Ion Batteries. Advanced Materials Interfaces, 7(2001022), 1-11 (2021)

[21] S.J. Lee, S.H. Lee, H.W. Kang, S. Nahm, B.H. Kim, H. Kim, S.H. Han: Flexible electrochromic and thermochromic hybrid smart window based on a highly durable ITO/graphene transparent electrode. Chemical Engineering Journal, 416(129028), 1-8 (2021)

[22] H.T. Kim, S.K. Jung, S.Y. Lee: Properties of ITO films deposited on paper sheets using a lowfrequency $(60 \mathrm{~Hz})$ DC-pulsed magnetron sputtering method. Vacuum, 187(110056), 1-5 (2021)

[23] L. Wen, B.B. Sahu, G.Y. Yeom, J.G. Han: Room temperature deposition of very thin and flexible crystalline ITO thin film using 3-D facing-magnetron sputtering plasma source. Vacuum, 193(110520), 1-5 (2021)

[24] H. Taha, K. Ibrahim, M.M. Rahman, D.J. Henry, C.Y. Yin, J.P. Veder, A. Amri, X. Zhao, Z.T. Jiang: Sol-gel derived ITO-based bi-layer and tri-layer thin film coatings for organic solar cells applications. Applied Surface Science, 530(147164), 1-7(2020) 